\title{
Inequality Measures for Wealth Distribution: Population vs Individuals Perspective
}

\author{
R. Pascoal ${ }^{\mathrm{a}}, \mathrm{H}$. Rocha $^{\mathrm{a}, \mathrm{b}, *}$ \\ ${ }^{a}$ CeBER \& FEUC, Av. Dias da Silva 165, 3004-512 Coimbra, Portugal. \\ ${ }^{b}$ INESC-Coimbra, Rua Silvio Lima, Polo II, 3030-290 Coimbra, Portugal.
}

\begin{abstract}
Economic inequality is, nowadays, frequently perceived as following a growing trend with impact on political and religious agendas. However, there is a wide range of inequality measures, each of which pointing to a possibly different degree of inequality. Furthermore, regardless of the measure used, it only acknowledges the momentary population inequality, failing to capture the individuals evolution over time. In this paper, several inequality measures were analyzed in order to compare the typical single time instant degree of wealth inequality (population perspective) to the one obtained from the individuals' wealth mean over several time instants (individuals perspective). The proposed generalization of a simple addictive model, for limited time average of individual's wealth, allows us to verify that the typically used inequality measures for a given snapshot instant of the population significantly overestimate the individuals' wealth inequality over time. Moreover, that is more extreme for the ratios than for the indices analyzed.
\end{abstract}

Keywords: Inequality Measures, Wealth Distribution, Econophysics

\section{Introduction}

Economic inequality plays, nowadays, an important role in both political and religious agendas. During the 2016 US presidential campaign, this topic was raised and debated more than once. Recently, while visiting Bolivia, Pope Francis said: "Working for a just distribution of the fruits of the earth and human labor is not mere philanthropy. It is a moral obligation." Despite economic inequality being a certainty, there is no consensus on the best way to measure it. There are inequality measures based on income, where the underlying idea is that what matters is the ability to attain a living standard by means of its own resources [1]. On the other hand, inequality measures based on consumption, claim that the present standard of living is the most important as compared

\footnotetext{
*Corresponding author. Tel.: +351 239 790527; fax: +351 239403511.

Email addresses: ruiapsp@fe.uc.pt (R. Pascoal), hrocha@mat.uc.pt (H. Rocha)
} 
to the way it is attained [2]. Finally, despite often ignored, wealth distribution plays an important role on describing economic inequality [3]. Inequality measures based on each of these dimensions - income, consumption, and wealth shed light on economic inequality from different perspectives, and thus all are useful for understanding the big picture. Wealth is, in general, the most unequally distributed of the three, consumption the least [4]. In this study, wealth distribution is considered for discussing inequality measures and their pitfalls.

Wealth inequality is typically assessed by considering a snapshot of the wealth distribution at a given time instant. Regardless of the measure used, it only acknowledges the momentary population inequality. Thus, the evolution over time of a given inequality measure represents the population evolution but fails to capture the individual evolution over time. For two different time instants, a given inequality measure may be the same (for the population) but individual outcome may vary significantly. Thus, the wealth ordering of the individuals may vary drastically.

It is of the utmost interest to inquire, in the context of wealth distribution for a given population, the extent to which the value of several inequality measures is significantly changed when they are applied on the mean of each individual wealth, in the last set of $n>1$ time instants, instead of solely inspecting the (population) wealth inequality at the last $n^{\text {th }}$ time instant. Since, by taking the mean, the most extreme values in current wealth are expected to be attenuated, it's only reasonable to ascertain that the value for a certain inequality measure should diminish when it is based in the individuals' means. The fact that, under certain assumptions, wealth distribution seems to stabilize in equilibrium shapes can be misleading since individuals suffer constant variations from time to time, specially in the upper tail, and their ordering can be changed even though the macroscopic distribution of wealth may appear to remain unchanged. This could let us wondering if the focus should not shift from the significance of inequality between individuals in a single time instant (population) to the acknowledgment of individuals inequality over time or even to the perception of the proportion of individuals that remain below a minimum level of wealth for a long time, jeopardizing a reasonable standard of living. This issues will be explored in this paper.

Real data has been used to study inequality (see, e.g. [5, 6]). However, an obvious drawback of our approach is the difficulty of using real data. Legal and confidentiality issues turn it impossible to trace the path of each individual for a given time frame. An alternative is to resort to data provided by a given theoretical model, where the simulated wealth path of each individual is explicitly known. This enables the evaluation of the extent to which the inequality is effectively smaller at individuals level as compared to the traditional population level analysis. The fact that theoretical models often state explicitly the symmetry between agents accentuates the temporary character of each individual position in the distribution and the possibly elusive nature of measures based in the wealth at a single moment. On the other hand, this fact suggests that these models may departure from the perception that in real distributions, individual positions may be more persistent. 
A generalization of a simple addictive model is proposed for which we verify that, for limited time average of individual's wealth, the typically used inequality measures for a given snapshot instant of the population significantly overestimate the individual's wealth inequality over time.

The remainder of the paper is organized as follows. In the next section we present the wealth theoretical model used. In section three we describe the inequality measures considered. Computational tests are presented in section four. In the last section we have the conclusions.

\section{Wealth theoretical model}

Historically, the first study of income (and wealth) distribution was made by Pareto [7]. Pareto show that the power law is well fitted only in the upper tail. R. Gibrat assumed that wealth and income dynamics are based in multiplicative stochastic processes which results in the lognormal law [8]. This, however, is non-stationary. More recently, a variety of different models have been proposed considering monetary wealth (given the relative difficulty to measure nonmonetary wealth). In these models, individuals' wealth evolution is based mostly in pairwise transactions resulting in money transfer from one to another. The primal assumption made is that total money in the economy is constant and as such is a conserved quantity. That is, it plays in Economics a similar role to the one played by energy in Statistical Mechanics. These type of wealth distribution models were firstly proposed by Angle [9, 10, 11, 12, 13]. Many physicists have proposed similar-type models including Ispolatov, Krapivsky and Redner [14], Bouchaud and Mézard [15], Chakraborti and Chakrabarti [16] and Drăgulescu and Yakovenko [17].

In this study, we follow the model in Drăgulescu and Yakovenko [17], a simple model of a closed economic system where the total money is conserved and the number of economic agents is fixed. Let us assume that there are $N$ agents each of which with an initial amount of money equal to $M$. In this model, transactions between two agents consider that the quantity lost by one is equal to that earned by the other (money is locally conserved in each transaction). Thus, at a given time $t$, the transition equations for the exchange of wealth between a pair of agents are:

$$
\left\{\begin{array}{l}
x_{i}^{t}=x_{i}^{t-1}-\Delta_{m} \\
x_{j}^{t}=x_{j}^{t-1}+\Delta_{m},
\end{array}\right.
$$

where $\Delta_{m}=\epsilon \times M$, with $\epsilon$ a random uniform variate in $[0,1]$. Notice that if $x_{i}^{t-1}<\Delta_{m}$, i.e. if agent $i$ does not have enough money to pay agent $j$, then the transaction does not occur. In order to speed up the process, at each time (iteration) $t, N / 2$ transactions take place. This does not necessarily imply that transactions occur for every agent at a given time since each pair of agents is chosen randomly at a time, being randomly decided the "winner" and the "looser". The idea that there is a "winner" and a "looser" in each transaction seems strange at first sight from the perspective of economic reasoning: a 
transition is supposed to occur only as long as it is advantageous to both interveners. We are stating implicitly the assumption that monetary transfer is the counterpart to a transfer of economic goods that does not get meterialized in non-monetary wealth (assumed to be non-existent).

Starting from a uniform distribution (all agents have the same wealth), the Boltzmann-Gibbs distribution is obtained in equilibrium, i.e. the probability distribution of money is

$$
P(m)=M \times e^{-\frac{1}{M} * m} .
$$

For example, considering $N=5000$ agents and $M=1000$ monetary units, wealth distribution reaches the stationary shape displayed in Fig. 1, perfectly fitting the Boltzmann-Gibbs distribution. Fig. 1 also displays the Gini index for the time frame considered $(t=500)$. As expected, after the model reaches stationarity, the Gini index tends to the same value (0.5).

A new model, generalization of the previous addictive model, based on the concept of creativity is proposed, where creativity is seen as having mass repercussion. The creative agent is rewarded by each of the other agents for the benefits coming from the new technological, managerial or marketing innovation. These are relatively more rare events than trivial one-to-one transactions. As we will see, at least for a long time period, there is a sustained increase in wealth inequality. This seems to be in line with the mainstream idea, though not consensual, that there is currently an increasing wealth inequality trend [4]. On the other hand, facts such as the general decrease in prices resulting from technological progress may diminish the minimum threshold for a standard of living making the importance of the lower part of the distribution less stringent.

Let us further detail this model. At each time instant $t$, a creativity money exchange may occur, i.e. a randomly chosen agent may receive money from other agents due to its creativity production. The chance of a creativity event at each time instant is defined as a Bernoulli random number with probability $p$. It is reasonable to consider a small value for $p$ as it is not expected that creativity production worthing money exchange happens too often. Thus, in this novel model, apart from the exchange of wealth given by Eq. (1) that happens at each time instant, if a creativity event occurs at a given time $t$, the transition equations for the creativity exchange of wealth between the creative agent $x_{i}$, randomly selected, and each one of the remaining agents $x_{j}, j=1, \ldots, N, j \neq i$ are:

$$
\left\{\begin{array}{l}
x_{i}^{t}=x_{i}^{t-1}+\Delta_{m} \times \xi \\
x_{j}^{t}=x_{j}^{t-1}-\Delta_{m} \times \xi,
\end{array}\right.
$$

where $\Delta_{m}=\epsilon \times M$, with $\epsilon$ a random uniform variate in $[0,1]$, and $\xi$ is a factor between 0 and 1 in order to consider only a percentage of the "usual" money exchange. Fig. 2 displays the model behavior for the same time window as before, considering $p=0.1$ and $\xi=0.005$. The Gini index presents a "soft" increasing trend for this time window and the money distribution displays a longer tail, no longer being exactly fitted by the Boltzmann-Gibbs distribution since no equilibrium is reached. As $p$ tends to zero, the money distribution 


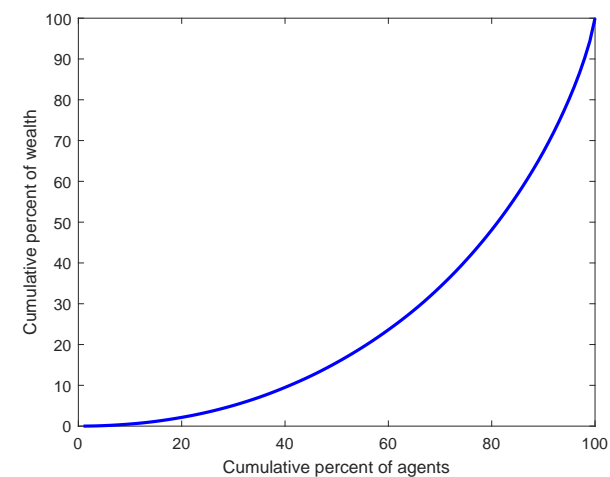

(a)
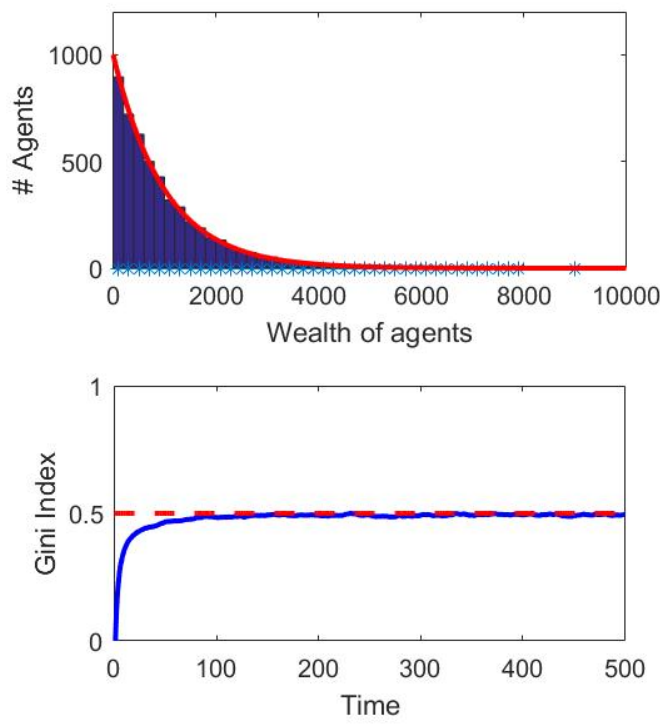

(b)

Figure 1: Cumulative distribution of agents vs wealth (a) and money distribution with corresponding Gini index evolution (b) for model corresponding to exchange of wealth given by Eq. (1). 


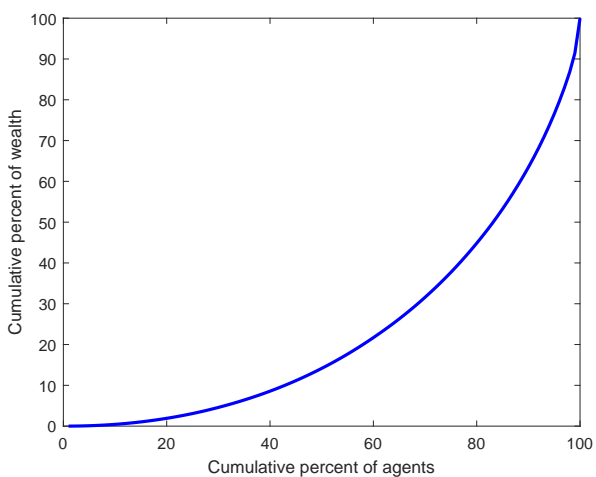

(a)
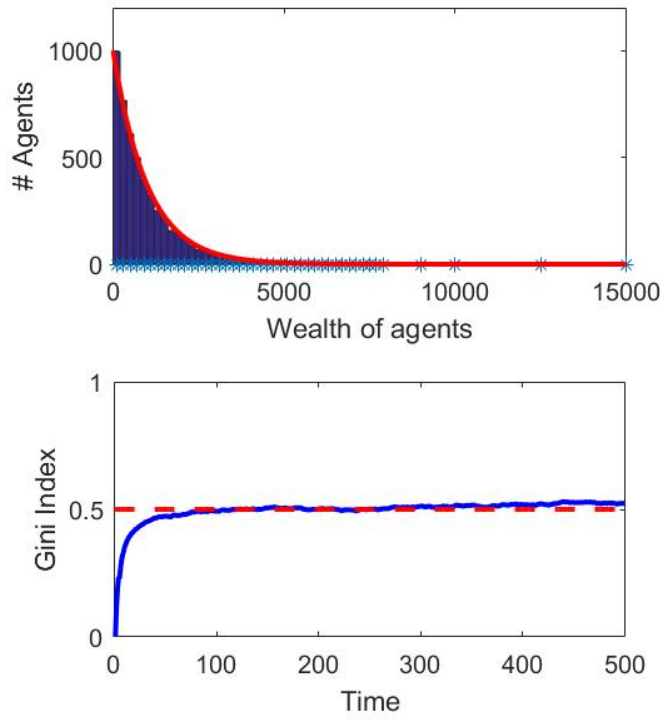

(b)

Figure 2: Cumulative distribution of agents vs wealth (a) and money distribution with corresponding Gini index evolution (b) for model corresponding to exchange of wealth given by Eqs. (1) and (2). 
tends to reach equilibrium, following the opposite path when $p$ increases. When $\xi$ increases, the length of the money distribution tail increases, as well as inequality. The thorough discussion of this particular model is out of the scope of this work, since here it serves the purpose of furnishing access to individual data across time allowing to evaluate the subsequent correction on inequality measure resulting from taking the (individuals) mean over time. Regardless of the model chosen, the limitation of fictitious (non-real) wealth distributions will always exist.

\section{Inequality Measures}

In this study, four inequality measures were chosen, two indices and two ratios: the Gini index, the Hoover (or Robin Hood) index, the 20:20 ratio, and the Palma ratio. Other indices could be of interest as the recently introduced Kolkata index [18]. All inequality measures considered have a corresponding representation in the Lorenz curve [19]. Fig. 3 illustrates such a curve for wealth distribution, plotting the share of population against the share of wealth. The horizontal axis displays the cumulative percentage of population ranked from the poorest to the wealthiest individual while on the vertical axis we have the corresponding cumulative percentage of total wealth. The Lorenz curve is a simple tool to learn the percentage of wealth that a given per cent of the population holds. E.g., for the given illustration, by simple inspection we verify that $90 \%$ of the population holds $60 \%$ of the total wealth or, in other words, the $10 \%$ wealthiest individuals hold $40 \%$ of the total wealth. The 45 -degree line represents equality of wealth for all individuals. Thus, the more distant the Lorenz curve is with respect to the equality line, the more unequal the wealth distribution.

\subsection{Indices}

The Gini index is the most widely cited inequality measure and very often used in official reports. It is computed as the ratio between the inequality gap area (painted area in Fig. 3) to the area of the triangle beneath the equality line. It ranges between 0 (perfect equality - no painted area) and 1 (wealthiest individual holds all wealth - painted area corresponds approximately to the triangle beneath the equality line). Thus, a higher Gini index corresponds to a more unequal distribution. An obvious limitation of this index is that completely different Lorenz curves, and thus inequalities with distinct meanings, may correspond exactly to the same Gini index value (as long as the inequality gap area is the same). Remark that in this index, as well as in the next, empirical implementation implies the use of discretization: the cumulation of frequency and wealth is done individual by individual.

The Hoover index corresponds to the proportion of total wealth that has to be redistributed in order to obtain perfect equality. It can be calculated as the ratio between the sum of excesses over the mean of individuals wealth and the total wealth. Graphically, it can be represented by the longest vertical 


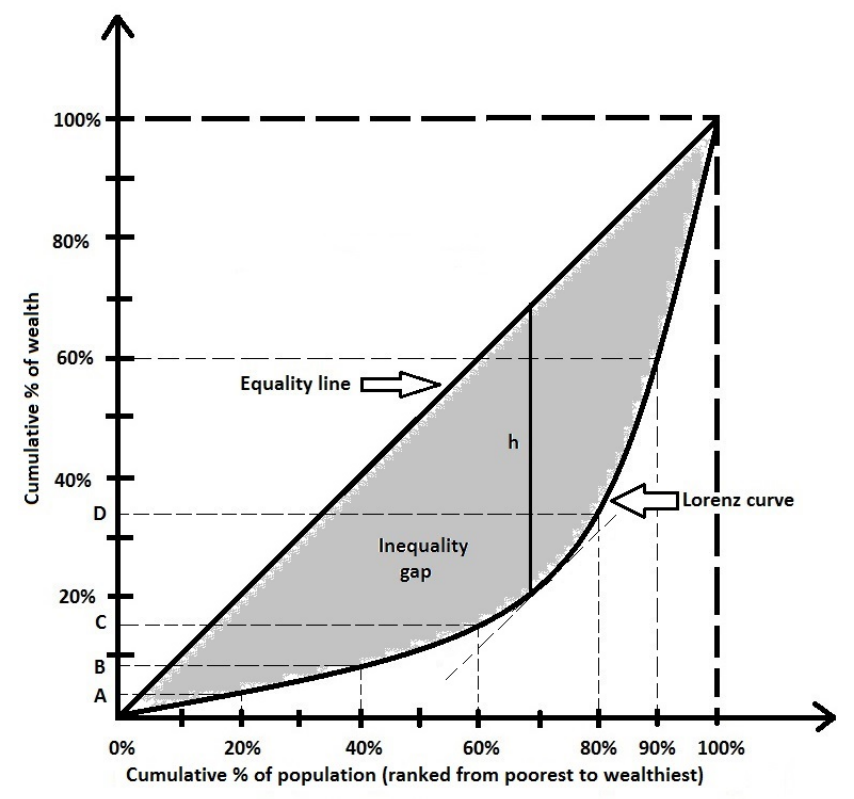

Figure 3: Illustration of Lorenz curve.

distance between the equality line and the Lorenz curve, which corresponds to $\mathbf{h}$ in Fig. 3. It takes values in the interval $[0,1)$. In case of perfect equality, Hoover index equals 0 since there is nothing to redistribute (h vanishes). If the wealthiest individual holds all wealth, all wealth but the mean must be redistributed and the ratio is close to one ( $\mathbf{h}$ is maximum and corresponds to the edge opposite to the equality line on the triangle beneath this 45-degree line). Thus, a higher Hoover index corresponds to a more unequal distribution. The limitation pointed to Gini index holds here.

\subsection{Ratios}

Some of the most simple and informative inequality measures are ratios. They present several advantages including simple calculation and intuitive interpretation. Decile dispersion ratios, where the 20:20 ratio and the Palma ratio are included, are the most used for inequality measures.

The 20:20 ratio compares the wealth of the $20 \%$ wealthiest individuals with the wealth of the $20 \%$ poorest individuals of the population. It displays the wealth of the rich as a multiple of the poor's wealth. It is used in official reports by the European Union [20] or by the United Nations [21]. For the illustration displayed in Fig. 3 , the $20: 20$ ratio equals $\frac{100-D}{A}$, where $A$ and $D$ are the quantiles of order 0.2 and 0.8 , respectively.

The Palma ratio compares the share of wealth of the $10 \%$ wealthiest individuals of the population's with the share of wealth of the $40 \%$ poorest [22]. 
Palma based this index on his empirical finding that the sum of shares of these two groups is equal to the share of the remaining group identified broadly as middle class. Furthermore, Palma observed that the difference of distribution over time is mostly the result of changes in the first two groups, the so-called "tails" of the distribution, while the share of wealth of the third group tends to be more stable [23]. For the illustration displayed in Fig. 3, the Palma ratio equals $\frac{100-60}{B}=\frac{40}{B}$, where $B$ is the quantile of order 0.4 . Since $B<10 \%$, this decile ratio allows the straightforward claim that the wealth of the $10 \%$ wealthiest is more than four times the wealth of the poorest $40 \%$.

Appropriate generalizations will be defined by considering different levels of the lower quantiles beyond which the individuals are considered to be below a minimum threshold for a standard of living.

\section{Computational Tests}

Our tests were performed on a 2.60Ghz Intel Core i7-6700HQ PC with 16 GB RAM and we used MATLAB (R2016a). For the creativity model presented in section 2, a large number of simulations was performed considering exactly the same model parameters used for obtaining the wealth distribution of Fig. 2. Inequality measures described in section 3 were then computed both considering the wealth distribution available at last time instant $t$ and the wealth distribution considering the individuals means of the last 2, 3, 5, 10, 20, 50 and 100 time periods. The means of these results over the simulations were computed as a Monte Carlo-like estimator. Comparisons were performed to evaluate the reduction on the measures when applied on the individuals means.

From simple inspection of Figs. 4 to 7, it is straightforward to conclude that the typically used inequality measures for a given snapshot instant of the population overestimate individuals' wealth inequality over time. Furthermore, that divergence is more extreme for ratios than for indices as depicted in Table 1. Comparing the two ratios, Palma ratio presented less differences comparing population and individuals perspectives, i.e. comparing the wealth distribution available at last instant $t=500$ and the wealth distribution considering the individual means of the last 2, 3, 5, 10, 20, 50 and 100 time periods. This observation is of the utmost relevance as the 20:20 ratio is widely used by international institutions as mentioned in section 2 .

As for measures based on quantiles of the wealth distribution, a natural generalization is to make the quantile order vary over an interval. When considering ratios between the 20:20 ratio and Palma ratio (40:10) by increasing the lower quantile order of the 20:20 ratio by two units at a time and decreasing the upper quantile order one unit at a time, the inequality measures obtained follow a decreasing path as well as the relative difference between population and individuals based measures. This observation corroborates Palma's empirical finding of a more stable middle class group for lower and upper parts of the distribution corresponding to the $40 \%$ poorest and $10 \%$ wealthiest.

Socially, it is an undesirable feature that a significant proportion of the population remains for a long period systematically below a low level of wealth. So, 


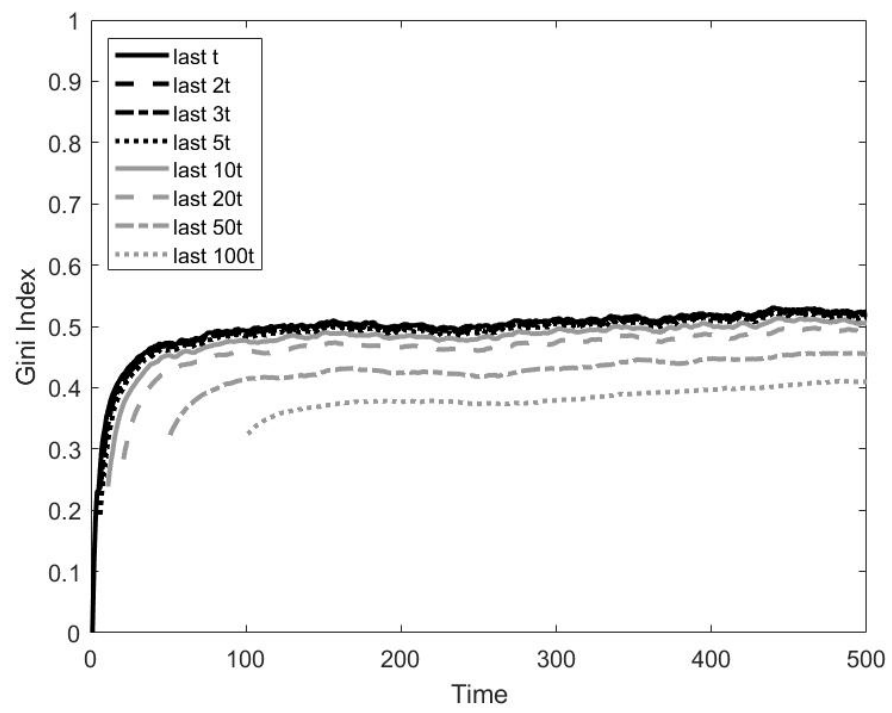

Figure 4: Gini index evolution over time considering the wealth distribution available at last instant $t$ and the wealth distribution considering the individuals means of the last $2,3,5,10$, 20, 50 and 100 time periods.

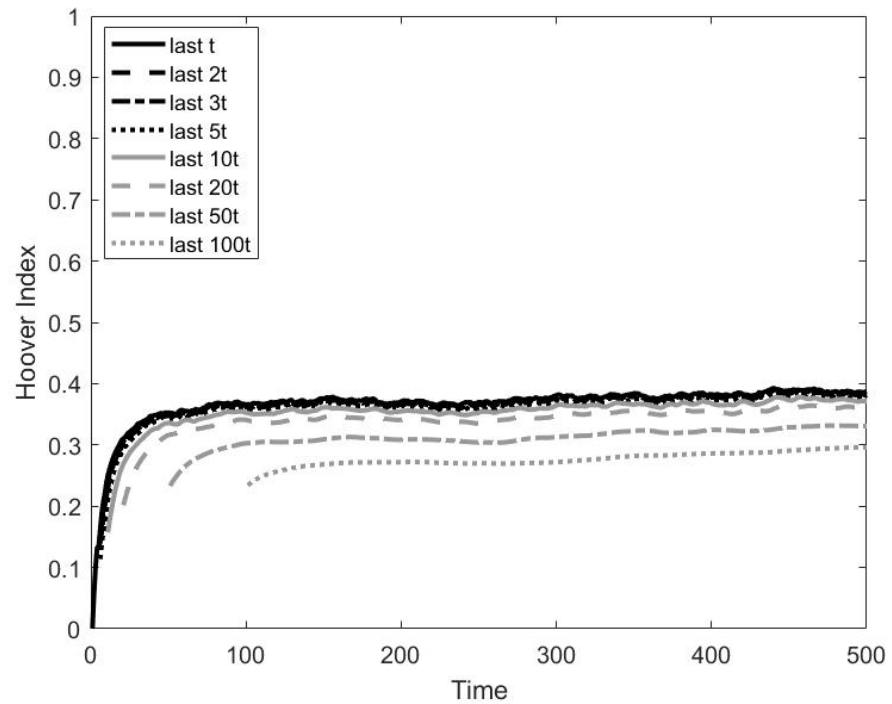

Figure 5: Hoover index evolution over time considering the wealth distribution available at last instant $t$ and the wealth distribution considering the individuals means of the last 2,3 , 5, 10, 20, 50 and 100 time periods. 


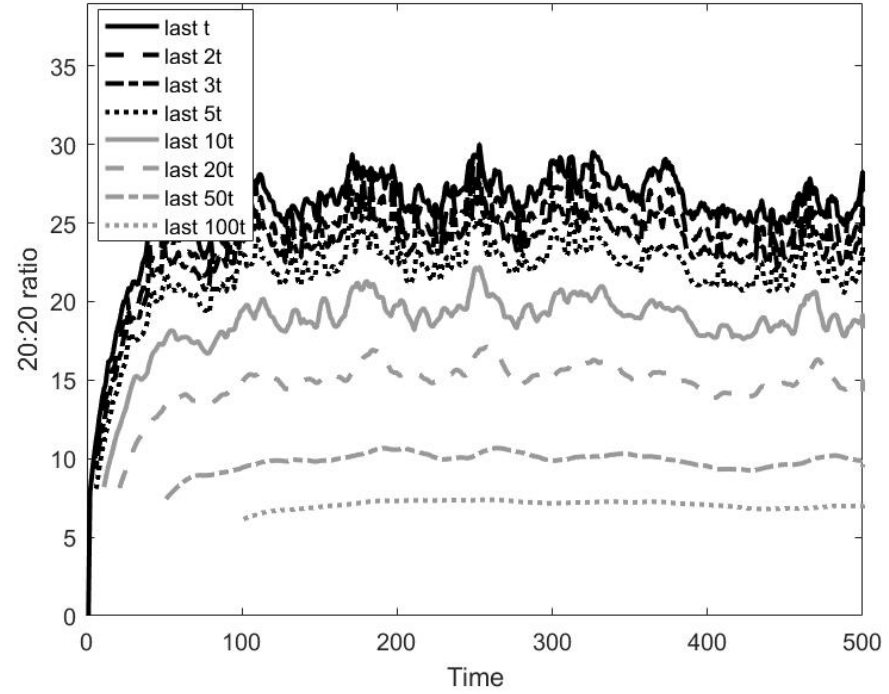

Figure 6: 20:20 ratio evolution over time considering the wealth distribution available at last instant $t$ and the wealth distribution considering the individuals means of the last $2,3,5,10$, 20, 50 and 100 time periods.

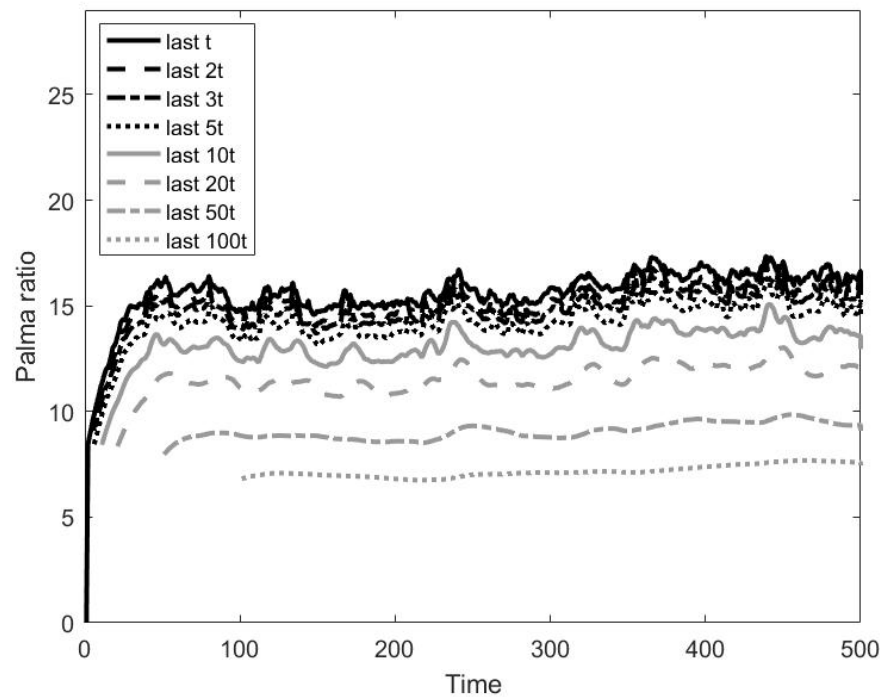

Figure 7: Palma ratio evolution over time considering the wealth distribution available at last instant $t$ and the wealth distribution considering the individuals means of the last $2,3,5,10$, 20,50 and 100 time periods. 
Table 1: Indices and ratios values considering the wealth distribution available at last instant $\mathrm{t}=500$ (last $\mathrm{t}$ ) and the wealth distribution considering the individuals means of the last 2,3 , 5, 10, 20, 50 and 100 time periods. For each measure, per cent comparison to last $\mathrm{t}$ is provided in a second column.

\begin{tabular}{|c|c|c|c|c|c|c|c|c|}
\hline & \multicolumn{2}{|c|}{ Gini index } & \multicolumn{2}{|c|}{ Hoover index } & \multicolumn{2}{|c|}{$20: 20$ ratio } & \multicolumn{2}{|c|}{ Palma ratio } \\
\hline & value & $\%$ last $\mathrm{t}$ & value & $\%$ last $\mathrm{t}$ & value & $\%$ last $\mathrm{t}$ & value & $\%$ last $\mathrm{t}$ \\
\hline last $\mathrm{t}$ & 0,524 & 100,0 & 0,388 & 100,0 & 28,261 & 100,0 & 17,962 & 100,0 \\
\hline last $2 \mathrm{t}$ & 0,521 & 99,3 & 0,385 & 99,2 & 27,863 & 98,6 & 17,712 & 98,6 \\
\hline last $3 t$ & 0,518 & 98,8 & 0,382 & 98,5 & 26,536 & 93,9 & 17,331 & 96,5 \\
\hline last 5t & 0,514 & 98,0 & 0,378 & 97,3 & 23,934 & 84,7 & 16,598 & 92,4 \\
\hline last $10 \mathrm{t}$ & 0,505 & 96,3 & 0,371 & 95,6 & 20,285 & 71,8 & 15,056 & 83,8 \\
\hline last $20 \mathrm{t}$ & 0,491 & 93,6 & 0,359 & 92,5 & 15,287 & 54,1 & 13,004 & 72,4 \\
\hline last $50 \mathrm{t}$ & 0,454 & 86,6 & 0,331 & 85,2 & 10,361 & 36,7 & 10,113 & 56,3 \\
\hline last $100 \mathrm{t}$ & 0,410 & 78,1 & 0,296 & 76,2 & 7,477 & 26,5 & 8,100 & 45,1 \\
\hline
\end{tabular}

we let this wealth threshold vary in a grid of values and observe the proportion of individuals for which the mean wealth over a period remains below that threshold. The wealth threshold ranges from the mean value $(M=1000)$ to zero and the dimension of the set of periods ranges from 0 to 100 time instants over which the mean is computed (see Fig. 8). It is seen that, as expected, for the last time instant $(t=0)$, the proportion of population with mean wealth below a given wealth threshold decays as the threshold tends to zero, but that proportion also decays when the number of times increases (individuals means are considered instead of a population snapshot).

Instead of a proportion of individuals having average wealth below a given wealth threshold, one can consider the proportion of individuals with wealth always below the given threshold during a time period. As expected, this proportion decays much faster than the proportion obtained by considering the mean wealth (see Fig. 9).

As a less stringent constraint, we lessen the number of times that individuals' wealth may be below a given threshold (it doesn't have to be always) and let this number vary in the interval between zero and the number of time instants considered. Fig. 10 displays the proportion of individuals that stays bellow a given wealth threshold for a number of time instants. For a wealth threshold equal to the mean value $(M=1000)$, the modal time is about 100 time instants (the maximum number of times considered). As the wealth threshold decreases, the modal time decreases as well, becoming zero for a threshold value equal (or inferior) to $20 \%$ of the mean wealth (200). This implies that individuals stay shorter time periods below lower wealth thresholds.

\section{Conclusions}

Several inequality measures were analyzed (Gini index, Hoover index, 20:20 ratio and Palma ratio) in order to compare the single time instant degree of wealth inequality to the one obtained from the (individuals) mean of several time instants. This comparison is made for a generalization of an addictive 


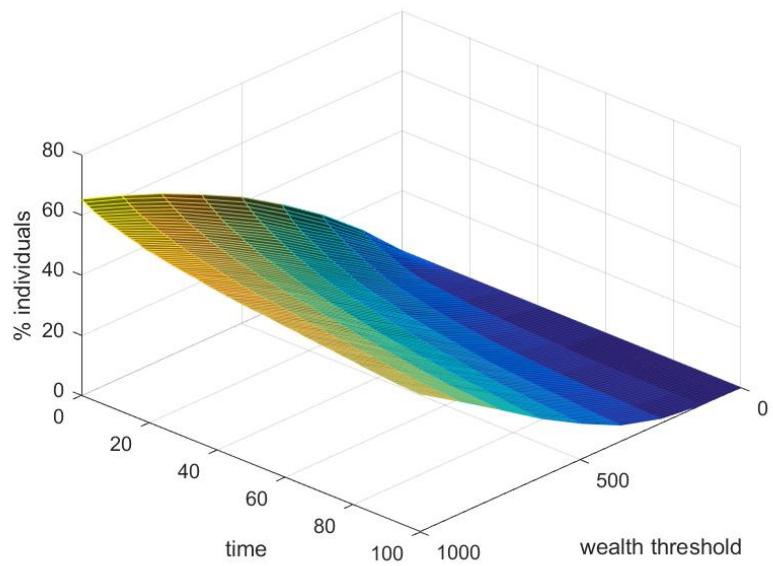

Figure 8: Surface representing the proportion of population under a given wealth threshold, in average, considering the last $\mathrm{t}(=100)$ time instants.

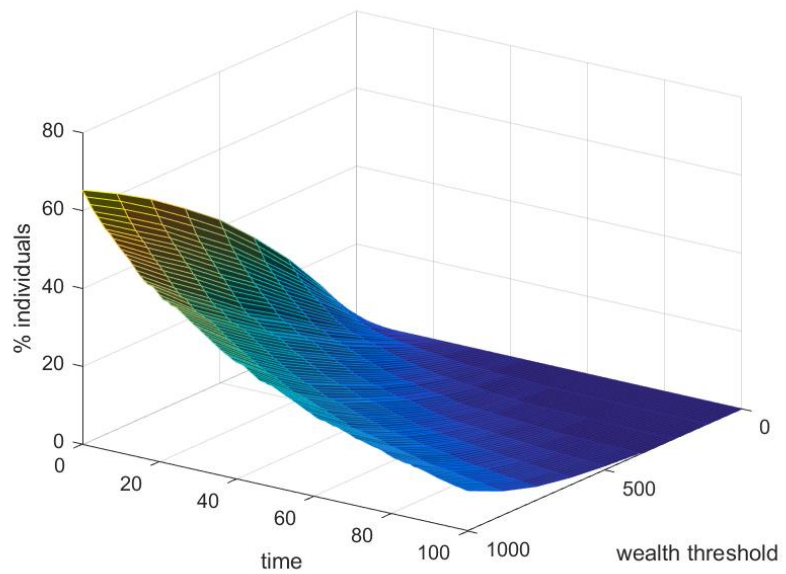

Figure 9: Surface representing the proportion of population always under a given wealth threshold from time 0 , the last temporal instance considered, up to the time they overcome the given wealth threshold. 


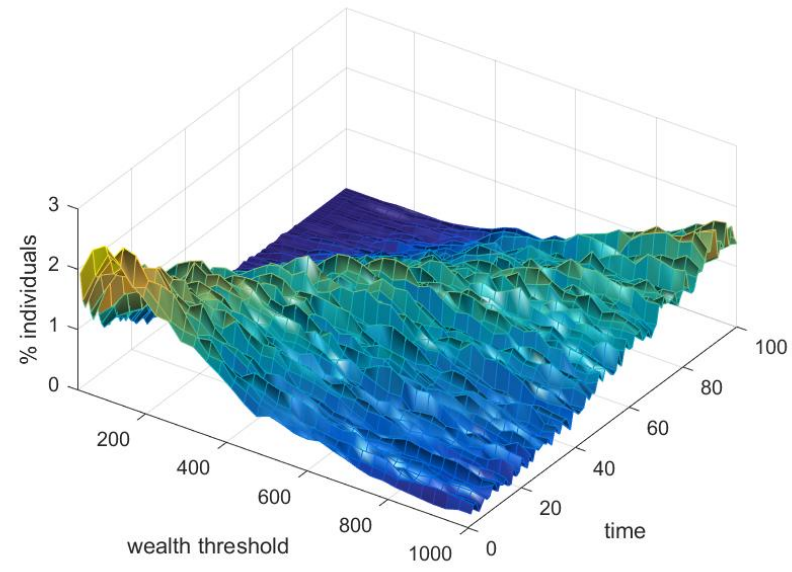

Figure 10: Surface displaying the proportion of population under a certain wealth threshold a number of times $t$.

model of Drăgulescu and Yakovenko [17] based on the transaction between pairs of individuals. The generalization accounts for creativity materialized in goods and services benefiting all the economy. As such, this is reflected in rare innovation for which its author is rewarded by the other agents with a substantial aggregated premium.

It should be highlighted that long-time average of individual's wealth will make the Lorenz curve collapse on the equality line for the addictive model of Drăgulescu and Yakovenko [17] as well as for our generalization and for all the remaining models mentioned in the paper. A noticeable exception of a statistical physics model where this collapse of the Lorenz curve to the equality line (for "sufficiently long time" average of individual's wealth) will not occur will be the Chatterjee-Chakrabarti-Manna (CCM) model, where the saving propensity of each individual is different $[24,25,26]$. Preliminary simulations with the CCM model, for limited time average of individuals wealth, lead to similar conclusions on the reduction of inequality measures when means are taken into account, especially for ratios.

The creativity model presented a slight increase in distribution inequality for the parameters considered during the time period in analysis. On the other hand, given the assumption of conservation of the total wealth combined with symmetry among agents, inequality can't grow indefinitely. In this model, the Gini index has a decrease that attains about $22 \%$ for the mean over 100 time instants while for the 20:20 ratio this decrease is amplified to some amazing $73.5 \%$. In other words, the 20:20 ratio at the last snapshot of the population is about four times greater than the 20:20 ratio considering the individuals means over 100 time instants. Regardless of the difficulty on defining the most 
appropriate time period, it is obvious that the population perspective (single instant) overestimates inequality as compared to the individuals perspective (over time). Furthermore, that effect has different magnitudes for different measures. Compared to indices, ratios presented larger differences between population and individuals perspectives of inequality. This is particularly amplified for the 20:20 ratio, a widely reported inequality measure by international institutions, which should be taken into account when interpreting or taking conclusions/decisions based on this inequality measure.

When considering the individuals wealth evolution over time, an informative measure is the proportion of individuals that falls bellow a given wealth threshold for a long time period. As the wealth threshold gets higher, the proportion of the individuals that falls below it a significant number of times tends to rise. An alternative focus to inequality could be obtained by constructing a measure that summarizes the pattern observed for the distribution of individuals over the binomial time/wealth threshold. However, defining an appropriate measure will certainly require further research that is beyond the scope of this work.

\section{Acknowledgements}

This work has been supported by the Fundação para a Ciência e a Tecnologia (FCT) under project grant UID/MULTI/00308/2013.

\section{References}

[1] E. Dabla-Norris, K. Kochhar, F. Ricka, E. Tsounta, Causes and Consequences of Income Inequality: A Global Perspective, IMF (2015).

[2] K.A. Hassett, A. Mathur, A New Measure of Consumption Inequality, American Enterprise Institute (2012).

[3] M.I. Norton, D. Ariely, Building a Better America - One Wealth Quintile at a Time, Perspectives on Psychological Science 6 (2011) 9-12.

[4] T. Piketty, Capital in the Twenty-first Century, Cambridge, Massachusetts: Harvard University Press (2014).

[5] M. Jagielski, K. Czyzewski, R. Kutner, H.E. Stanley, Income and wealth distribution of the richest Norwegian individuals: An inequality analysis, Physica A 474 (2017) 330-333.

[6] B. Oancea, T. Andrei, D. Pirjol, Income inequality in Romania: The exponential-Pareto distribution, Physica A 469 (2017) 486-498.

[7] V. Pareto, Cours d'Economie Politique, F. Pichon Imprimeur-Editeur, Lausanne and Paris (1897).

[8] R. Gibrat, Les Inégalités économiques, Recueil Sirey, Paris (1931). 
[9] J. Angle, Coalitions in a stochastic process of wealth distribution, Proceedings of the American Statistical Association, Social Statistics Section. Alexandria, VA: American Statistical Association (1986) 259-263.

[10] J. Angle, The Inequality Process and the distribution of income to blacks and whites, Journal of Mathematical Sociology 17 (1992) 77-98.

[11] J. Angle, Deriving the size distribution of personalwealth from 'the rich get richer, the poor get poorer', Journal of Mathematical Sociology 18 (1993) $27-46$.

[12] J. Angle, How the gamma law of income distribution appears invariant under aggregation, Journal of Mathematical Sociology 21 (1996) 325-358.

[13] J. Angle, The Inequality Process as a wealth maximizing process, Physica A 367 (2006) 388-414.

[14] S. Ispolatov, P.L. Krapivsky, S. Redner, Wealth distributions in asset exchange models, Eur. Phys. J. B 2 (1998) 267-276.

[15] J.P. Bouchaud, M. Mézard, Wealth condensation in a simple model of economy, Physica A 282 (2000) 536-545.

[16] A. Chakraborti, B.K. Chakrabarti, Statistical mechanics of money: how saving propensity affects its distribution, Eur. Phys. J. B 17 (2000) 167-170.

[17] A. Drăgulescu, V.M. Yakovenko, Statistical mechanics of money, Eur. Phys. J. B 17 (2000) 723-729.

[18] A. Chatterjee, A. Ghosh, B.K.Chakrabarti, Socio-economic inequality: Relationship between Gini and Kolkata indices, Physica A 466 (2017) 583-595.

[19] M.O. Lorenz, Methods of measuring the concentration of wealth, Publications of the American Statistical Association 9 (1905) 209-219.

[20] Eurostat, S80/S20 income quintile share ratio, http://ec.europa.eu/ eurostat/web/gdp-and-beyond/quality-of-life/s80s20-income-quintile. Retrieved 11 May 2017.

[21] United Nations Development Program, Human Development Report, New York: Oxford University Press (2004).

[22] J.G. Palma, Homogeneous middles vs. heterogeneous tails, and the end of the 'Inverted-U': the share of the rich is what it's all about, Cambridge Working Papers in Economics (CWPE) 1111 (2011).

[23] A. Cobham, L. Schlogl, A. Sumner, Inequality and the Tails: The Palma Proposition and Ratio Revisited, Department of Economic and Social Affairs Working Paper No. 143 (2015). 
[24] A. Chatterjee, B. K. Chakrabarti, S. S. Manna, Pareto law in a kinetic model of market with random saving propensity, Physica A 335 (2004) 155163.

[25] B. K. Chakrabarti, A. Chakraborti, S. R. Chakravarty, A. Chatterjee, Econophysics of Income and Wealth Distributions, Cambridge Univ. Press (2013).

[26] L. Pareschi, G. Toscani, Interacting Multiagent Systems, Oxford Univ. Press (2014). 